\title{
Isolation of a Highly Thermal Stable Lama Single Domain Antibody Specific for Staphylococcus aureus Enterotoxin B
}

\author{
Russell R Graef ${ }^{1}$, George P Anderson ${ }^{2 *}$, Katherine A Doyle ${ }^{1}$ Dan Zabetakis², Felicia N Sutton ${ }^{1}$, Jinny L Liu², \\ Joseline Serrano-González ${ }^{3}$, Ellen R Goldman² and Lynn A Cooper ${ }^{1 *}$
}

\begin{abstract}
Background: Camelids and sharks possess a unique subclass of antibodies comprised of only heavy chains. The antigen binding fragments of these unique antibodies can be cloned and expressed as single domain antibodies (sdAbs). The ability of these small antigen-binding molecules to refold after heating to achieve their original structure, as well as their diminutive size, makes them attractive candidates for diagnostic assays.

Results: Here we describe the isolation of an sdAb against Staphyloccocus aureus enterotoxin B (SEB). The clone, $A 3$, was found to have high affinity $(K d=75 \mathrm{pM})$ and good specificity for $S E B$, showing no cross reactivity to related molecules such as Staphylococcal enterotoxin A (SEA), Staphylococcal enterotoxin D (SED), and Shiga toxin. Most remarkably, this anti-SEB sdAb had an extremely high $\mathrm{Tm}$ of $85^{\circ} \mathrm{C}$ and an ability to refold after heating to $95^{\circ}$ C. The sharp Tm determined by circular dichroism, was found to contrast with the gradual decrease observed in intrinsic fluorescence. We demonstrated the utility of this $s \mathrm{AAb}$ as a capture and detector molecule in Luminex based assays providing limits of detection (LODs) of at least $64 \mathrm{pg} / \mathrm{mL}$.

Conclusion: The anti-SEB sdAb A3 was found to have a high affinity and an extraordinarily high Tm and could still refold to recover activity after heat denaturation. This combination of heat resilience and strong, specific binding make this sdAb a good candidate for use in antibody-based toxin detection technologies.
\end{abstract}

\section{Background}

The "Amerithrax" anthrax attacks of 2001 focused attention on the need for rapid and robust diagnostic methods to detect biological threat agents in environmental and clinical samples [1]. Many laboratory diagnostic platforms (eg enzyme linked immunosorbent assays [ELISAs], flow cytometry, and western blots) use targetspecific antibodies to detect microbial pathogens and toxins. Antibody based assays are particularly useful for identifying highly purified biological toxins because such samples contain little if any nucleic acids on which polymerase chain reaction (PCR) assays depend [2-4]. Simplified antibody-based tests (e.g. lateral flow assays) have been developed for field analysis and are used for a wide

\footnotetext{
* Correspondence: george.anderson@nrl.navy.mil; lynncooper@mitre.org 'MITRE Corporation, 7515 Colshire Drive, McLean, Virginia, 22102, USA ${ }^{2}$ Center for Bio/Molecular Science and Engineering, Naval Research Laboratory, 4555 Overlook Avenue SW, Washington, DC, 20375, USA Full list of author information is available at the end of the article
}

range of applications [5,6]. However the standard reagent-grade antibodies used in these tests are heat labile, meaning that they may degrade under harsh conditions, limiting field applications $[7,8]$. Replacing these standard antibodies with a type of immunoreagent that is more stable could greatly simplify the logistical demands of field-deployed biosensors.

A handful of animal species produce antibodies that are functional but are devoid of light chains. These heavy chain only antibodies (HcAbs) can be isolated from members of the Camelid family and from sharks $[9,10]$. The variable regions of $\mathrm{HcAb}\left(\mathrm{V}_{\mathrm{HH}}\right)$ when expressed as recombinant fragments, often called single domain antibodies (sdAbs), exhibit valuable characteristics including small size (12-16 kDa) and the ability to refold following heating to temperatures which normally causes the irreversible denaturation of conventional antibodies $[11,12]$. These properties make sdAbs attractive candidates for the development of immunodiagnostic
C Biomed Central

(c) 2011 Graef et al; licensee BioMed Central Ltd. This is an Open Access article distributed under the terms of the Creative Commons Attribution License (http://creativecommons.org/licenses/by/2.0), which permits unrestricted use, distribution, and reproduction in any medium, provided the original work is properly cited. 
tests [13]. Previously, sdAbs able to bind small molecules (caffeine and methotrexate), or toxins (botulinum, ricin, cholera, and scorpion), and viruses (rotavirus, HIV, Vaccinia, and Marburg) have been isolated [11,14-20]. Of particular relevance, a sdAb has recently been developed for the related toxin, toxic-shock syndrome toxin 1 (TSST-1),[21] and another for the detection of Staphylococcus aureus [22].

Staphylococcus aureus produces a number of potent enterotoxins, of which Staphylococcal enterotoxin B (SEB) is the most common cause of food borne poisoning. SEB is a single-chain polypeptide of 239 amino acids and has a molecular mass of $28.4 \mathrm{kDa}$ [23]. In addition to SEB's role in food poisoning, the toxin is considered a potential biological threat agent, and is listed as a category B select agent by the Centers for Disease Control. Here we describe the isolation and characterization of an anti-SEB single domain antibody from an immunized llama, and demonstrate its utility for detecting SEB in immunoassays.

\section{Results and Discussion}

\section{Evaluation of Serum and purified anti-SEB IgG}

Our goal was to generate camelid sdAbs against SEB using a phage display library derived from the white blood cells of a llama serially immunized with SEB toxoid and to detail the antigen binding properties of isolated anti-SEB sdAbs. The llama (Spode) was immunized using SEB toxoid, and the presence of antiSEB toxin antibodies in the plasma was verified by ELISA (Figure 1) prior to library construction. Once we confirmed a robust immune response towards SEB, we isolated RNA from the llama's white blood cells for library construction.

In addition to confirming the presence of anti-SEB antibodies in the llama plasma, the Immunoglobulin G (IgG) was purified and subclasses fractionated into conventional (IgG1) and heavy-chain only antibody (IgG2 and IgG3) fractions using Protein G and Protein A columns. The IgG subclasses were evaluated by fast protein liquid chromatography (FPLC). The IgG2 was clearly smaller than the IgG1 as seen by FPLC, and composed of only heavy chains as observed by polyacrylamide gel electrophoresis (PAGE) (data not shown) confirming the lack of light chains. However, the IgG3 fraction failed to separate from IgG1, thus results using this material are not shown. The lama polyclonal antibody and purified conventional (IgG1) and heavy chain only (IgG2) fractions were evaluated along with the isolated sdAb to assess their specificity and thermal stability. The llama polyclonal antibody was also paired with the isolated sdAb for use in sandwich immunoassays for the detection of SEB. These results are discussed below.

\section{Isolation of the anti-SEB SdAb A3}

A library was developed from the peripheral blood lymphocytes purified from the immunized llama. This library was estimated to have a size of $\sim 10^{6}$ by the number of transformants. Eleven individual clones were plaque purified and sequenced; all 11 were found to be unique and full length. To isolate a SEB specific sdAb from the phage display library we conducted 3 rounds of panning, followed by monoclonal phage ELISA. Following ELISA, 11 clones were selected and sequenced; all sequenced clones $(n=11)$ had identical base pair sequences indicating that by round 3 of panning the diversity of binders had been reduced to a single clone. The predicted amino acid sequence, represented by clone A3, is shown in Figure 2A. To evaluate its binding characteristics, clone A3 was expressed as a soluble protein and purified; its size $(\sim 16 \mathrm{kDa})$ was confirmed on a western blot (Figure 2B).

\section{Specificity of the anti-SEB SdAb A3 and polyclonal Ilama $\lg \mathrm{G}$}

The initial studies examined the cross-reactivity of A3, as well as both IgG1 and IgG2 subclass fractions, to a panel of common threat agent toxins using Luminex direct binding assays (Figure 3). We found that A3 was specific for SEB toxin and did not cross react with other toxin targets including super antigen relatives such as SEA and SED. This specificity, which is also observed in conventional monoclonal antibodies, is ideal for developing target-specific reagents for immunodiagnostics or environmental sensing. The llama polyclonal IgG1 and IgG2 subclass antibodies purified from the animal demonstrated a much higher titer for SEB relative to the other toxins, but at higher IgG concentrations they displayed cross reactivity towards SEA and SED, demonstrating the rationale for using highly selective monoclonal antibody reagents.

\section{Thermal stability of the anti-SEB SdAb A3 and polyclonal llama lgG}

Once the specificity of the sdAb A3 had been established we evaluated its thermal stability, comparing the selected sdAb with anti-SEB MAb 3b2a, as well as the llama polyclonal IgG1. First, an activity assay was utilized, wherein biotinylated aliquots of sdAb A3, llama polyclonal IgG, rabbit anti-SEB (RA-SEB) and anti-SEB $\mathrm{MAb} 3 \mathrm{~b} 2 \mathrm{a}$ were evaluated by heating to $85^{\circ} \mathrm{C}$ for various lengths of time. After cooling, their ability to bind SEB coated microspheres was evaluated (Figure 4). Within 10 minutes of heating conventional llama antibody (IgG1), as well as RA-SEB and MAb 3b2a, rapidly lost $80 \%$ of their binding activity and after an hour they had lost over $95 \%$. The binding activity of the llama heavy chain antibody subclass IgG2 initially declined $40 \%$ 


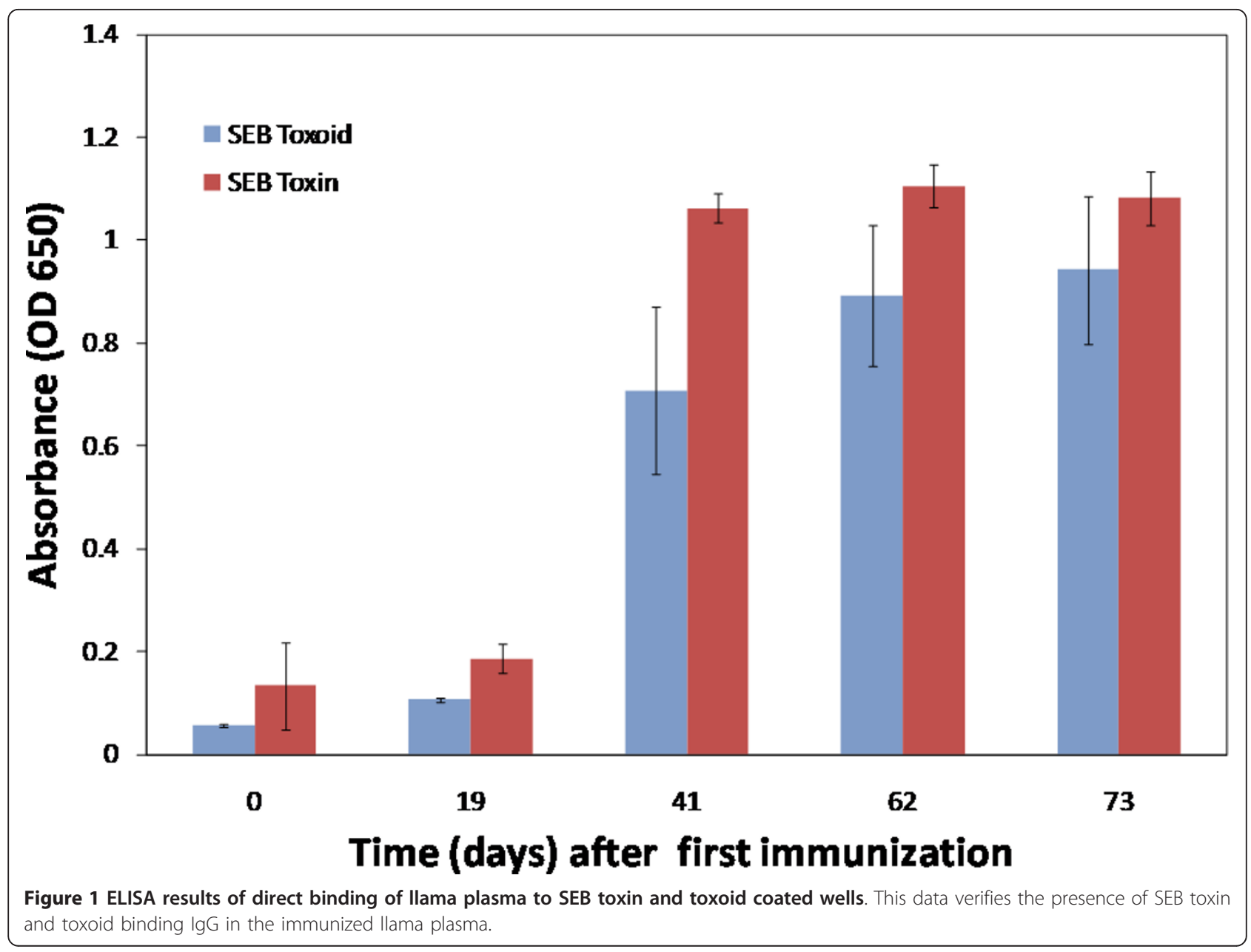

during the first 5 minutes of heating but then declined only slowly over the remaining forty five minutes, retaining $\sim 30 \%$ of the initial activity. Clearly, the sdAb A3 exhibited the best stability, decreasing slightly (20\%) upon initial heating but then residual activity remained fairly constant, dropping again during the last $15 \mathrm{~min}$ utes to $45 \%$ of the initial activity after heating for 1 hour.

Next, we examined the effect of heating on the secondary structure of the antibodies (non-biotinylated) by monitoring their $\mathrm{CD}$ spectrum during heating. The $\mathrm{CD}$ spectrum shows that the sdAb A3 unfolded upon heating and then refolded when cooled (Figure 5A). A3 was then subjected to multiple cycles of heating and cooling (Figure 5B and 5C). By monitoring the ellipticity at 202 $\mathrm{nm}, \mathrm{A} 3$ was observed to start losing secondary structure at $80^{\circ} \mathrm{C}$, with a melting point at $\sim 85^{\circ} \mathrm{C}$. After cooling down, the sdAb was found to recover the majority of its secondary structure. The unfolding and refolding transitions occurred rapidly and at nearly the same temperature. Clone A3 has one of the highest melting points we have observed to date for a sdAb [24-29]. To confirm this melting point, this value was also determined using DSC (Figure 6). This method yielded a melting point of $85^{\circ} \mathrm{C}$, however under these conditions, high concentration and high maximum temperature, no refolding was observed. To evaluate the melting temperatures of sdAbs, including A3, we constructed a normal probability plot (Figure 7). Points on this plot will lie along the regression line if the data conforms to a normal distribution. For 11 antibodies for which Tm has been determined (unpublished observations) the data can be seen to fit well to a normal distribution. A3, with a Tm above $85^{\circ} \mathrm{C}$, can be seen to deviate significantly, implying that this data cannot be explained as an expected variation in a normal distribution.

Another interesting finding was that even though the sdAb A3 maintains its secondary structure until $80^{\circ} \mathrm{C}$, its intrinsic fluorescence decreased in a nearly linear relationship with temperature (Figure $8 \mathrm{~A}$ and $8 \mathrm{~B}$ ). The decrease in fluorescence intensity can be attributed to the decreasing hydrophobic environment of buried tryptophans as well as to increased vibrational decay due to increased motion. Fundamentally, these results indicate 


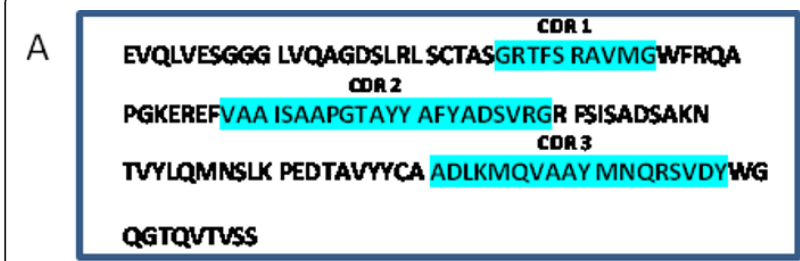

B

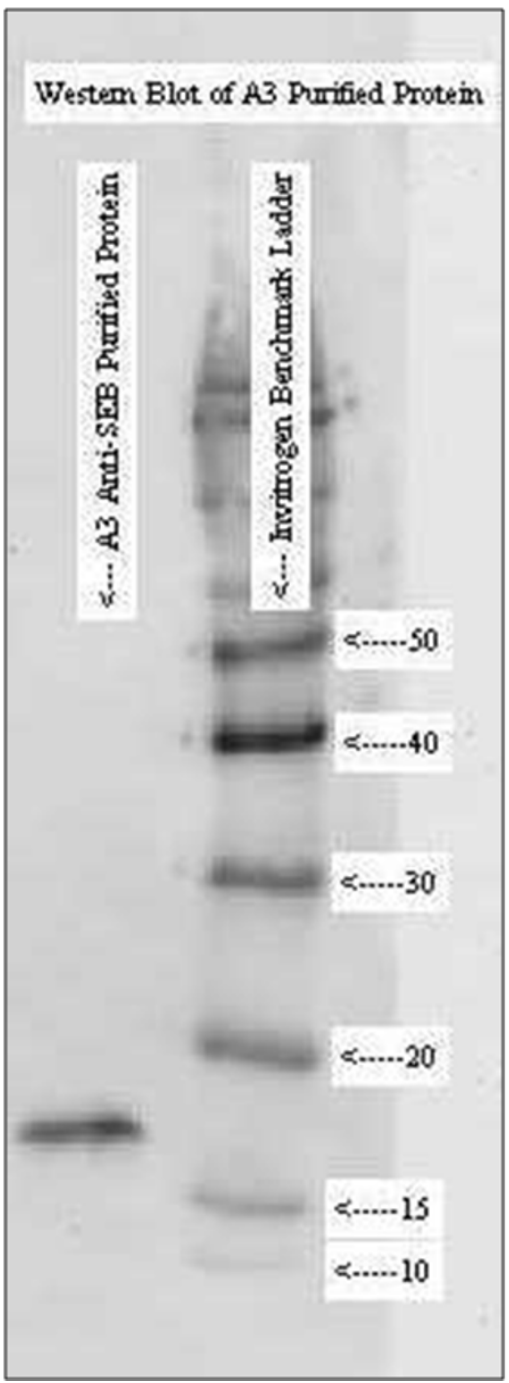

Figure 2 Amino acid sequence and western blot of $s d A b A 3$. A) Predicted amino acid sequence of the $\mathrm{A} 3$ anti-SEB sdAb. CDRs are highlighted. B) Western Blot of purified sdAb A3 using anti-His-HRP antibody. Benchmark ladder.

that for $\mathrm{sdAb}$, fluorescence intensity is a poor metric to monitor protein folding, with the actual CD spectra providing a more precise $\mathrm{Tm}$. These results are contrasted by the $\mathrm{CD}$ and fluorescence measurements of the llama IgG1 anti-SEB, which lost both secondary structure and fluorescence intensity at comparable rates (Figure $8 \mathrm{C}$ and $8 \mathrm{D}$ ). More importantly, conventional IgGs do not refold properly after heating,
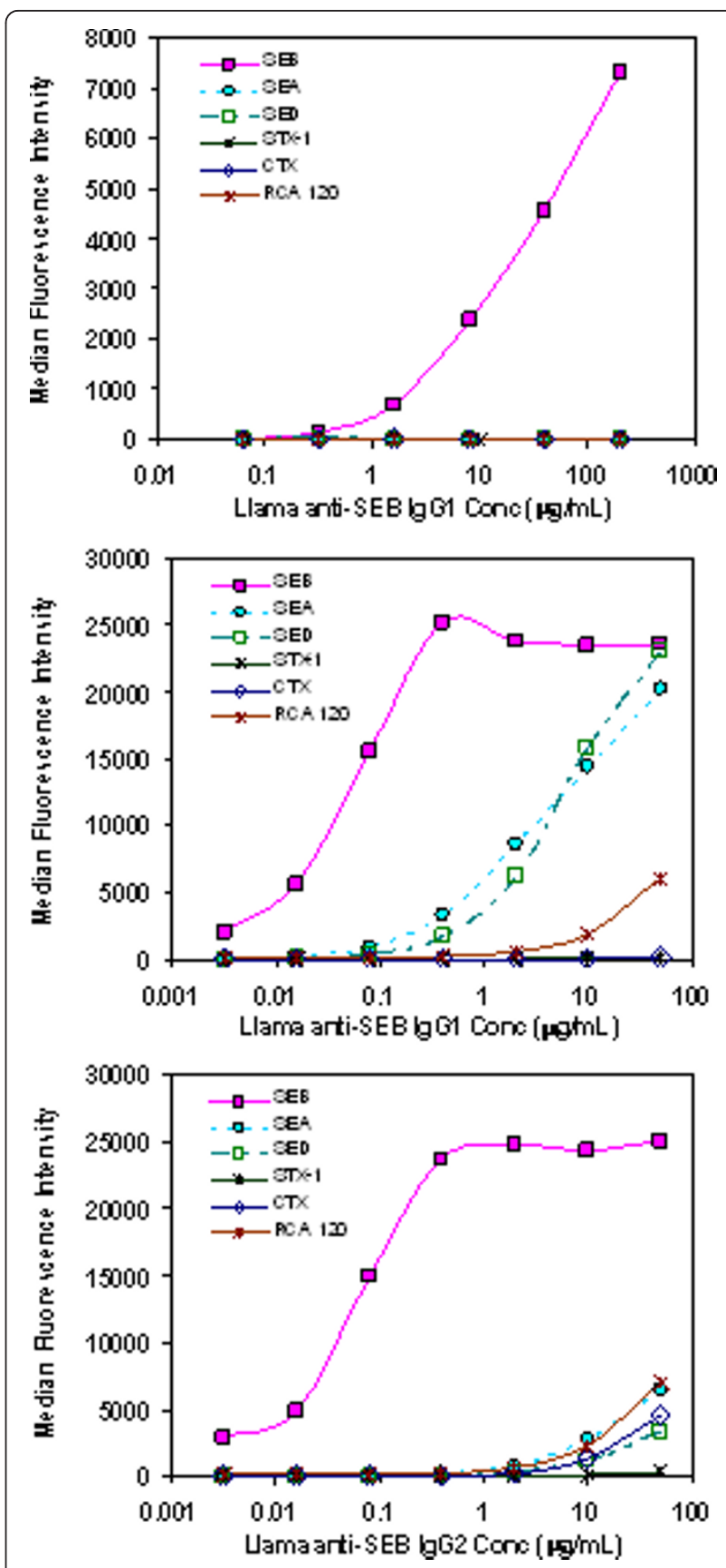

Figure 3 Evaluation of specificity by Luminex fluid array direct binding assays: Bt-sdAb A3 (top panel), Bt- Ilama anti-SEB lgG1 (middle panel), and Bt-lgG2 (bottom panel) subclass fraction. Result show A3 is highly specific for SEB.

permanently losing the bulk of their secondary structure and activity. The thermal resilience shown by the sdAb could be extremely useful for detection systems because sdAb molecules could be repeatedly used to capture their target simply by serially heating and cooling. Such an application would be an important contribution to current bio-detection technologies as it 


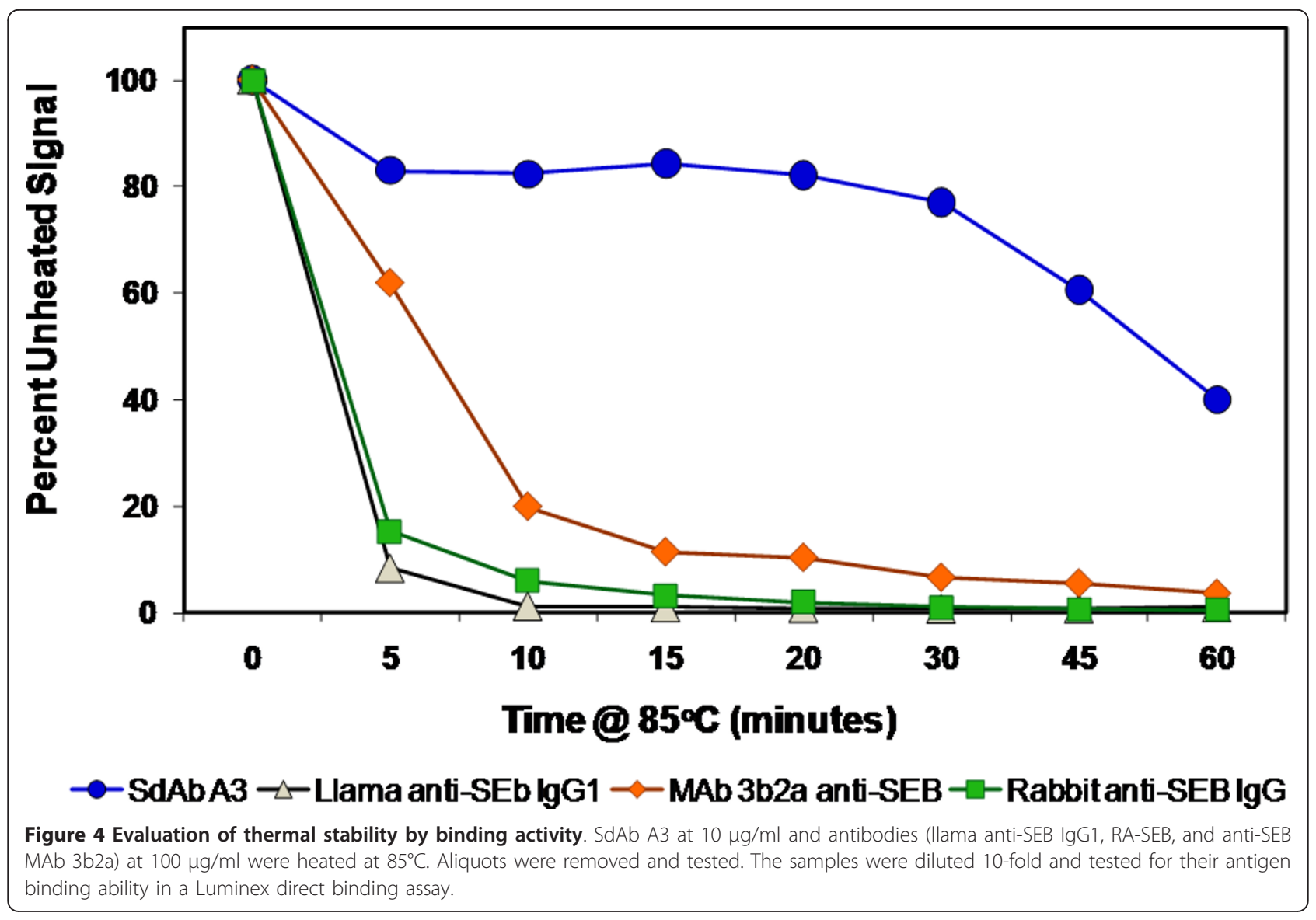

could greatly enhance the ability to field continuous monitoring systems.

\section{Affinity of the anti-SEB sdAb A3}

The affinity constants of sdAb A3 were determined using SPR (Figure 9). A3 was found to have a very high affinity for SEB, Table 1. Similar results were obtained from both the covalently immobilized A3 and the Bt-A3 bound to a NeutrAvidin coated chip, with both yielding a KD of $\sim 400$ to $600 \mathrm{pM}$. When the SEB was immobilized, A3 bound even more effectively, with a measured $\mathrm{KD}$ of $\sim 75 \mathrm{pM}$, confirming the high affinity interaction that A3 has for SEB. The MAb 3b2a also had a very high affinity for SEB. In fact it's off rate was so low we were unable to determine an accurate affinity (KD $\leq 100$ $\mathrm{pM})$. These high affinities explain the ability of these immunoreagent to sensitively detect SEB as demonstrated below.

Sandwich immunoassays for the detection of SEB using the anti-SEB sdAb A3

The final studies utilized the sdAb A3 as either the capture molecule or the detection molecule in fluid array sandwich immunoassays for SEB. In addition to A3, the
MAb 3b2a and the purified llama polyclonal anti-SEB were utilized (Figure 10). SdAb A3 functioned well when paired with MAb 3b2a in the sandwich immunoassay indicating that they recognize different epitopes. When A3 was the Bt detector and MAb 3b2a the capture molecule (Figure 10 top panel) the assay could detect as little as $64 \mathrm{pg} / \mathrm{mL}$ SEB. The reverse configuration, Bt-MA3b2a detector and sdAb A3 capture (Figure 10 middle panel), was not as sensitive with a LOD of 40 $\mathrm{ng} / \mathrm{ml}$. While the llama polyclonal antibody was a poor capture molecule, presumably due to its relatively low titer, the Bt-llama polyclonal antibody functioned very effectively as a Bt-labeled detector in combination with all the captures (Figure 10 bottom panel). Limits of detection of $64 \mathrm{pg} / \mathrm{mL}$ SEB were observed with the sdAb A3 and llama IgG1 anti-SEB captures, while when paired with the monoclonal it gave a signal intensity $\sim 10$-fold greater, suggesting that pair would have an even more impressive LOD. The large differential between the A3 and the Mab 3b2a capture molecules when using the polyclonal detector may occur because the polyclonal antibody favors the same epitope as the A3. Nonetheless, the A3 sdAb provided detection limits to $64 \mathrm{pg} / \mathrm{mL}$ as both a capture and detector molecule, 

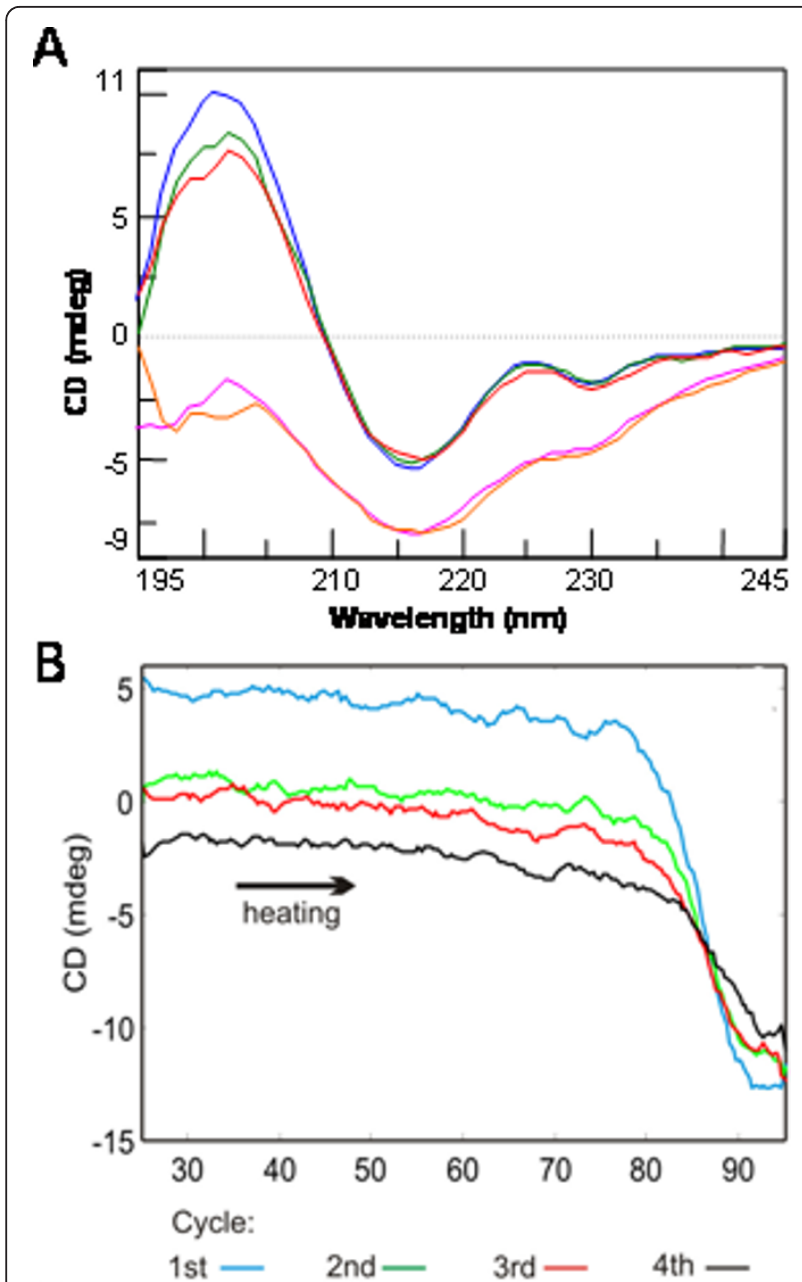

C

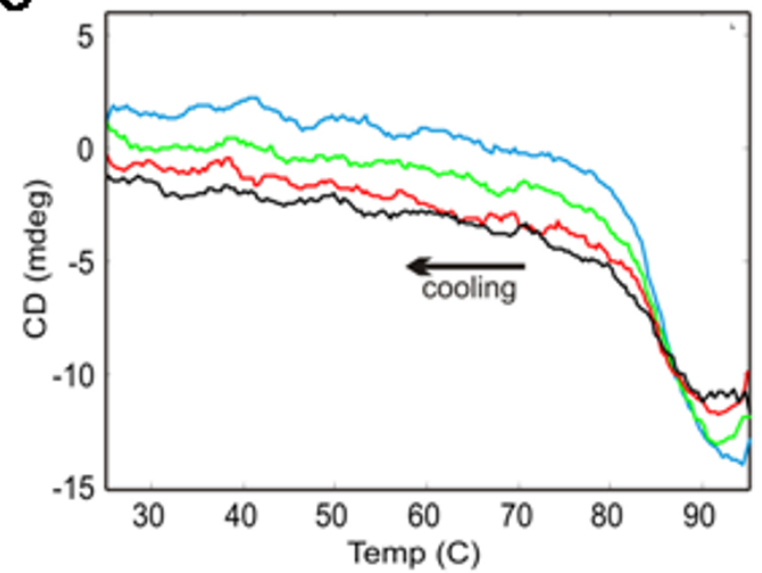

Figure 5 Evaluation of A3's thermal stability by monitoring secondary structure by $\mathrm{CD}$. A3 was heated to $85^{\circ} \mathrm{C}$ and then cooled to $25^{\circ} \mathrm{C}$ repeatedly: A) CD spectra of $\mathrm{A} 3$ (blue line) and upon heating to $85^{\circ} \mathrm{C}\left(1^{\text {st }}\right.$ cycle, pink; $2^{\text {nd }}$ cycle, orange) and upon cooling to $25^{\circ} \mathrm{C}\left(1^{\text {st }}\right.$ cycle, green line; $2^{\text {nd }}$ cycle, red line). Alternatively, secondary structure can be monitored and Tm determined by tracking the ellipticity change at single wavelength 202 nm: B) 4 heat cycles to $95^{\circ} \mathrm{C}$, first cycle blue, second cycle green, third cycle red, and fourth cycle black; C) 4 cooling cycles color coded as in panel B. which is more than adequate to meet most current detection requirements. In addition, the thermal stability and ability to re-fold after denaturation should give sdAbs, such as A3, an advantage when incorporated into biosensors for use in austere environments. For such applications it would be advantageous to isolate a second anti-SEB sdAb that recognized a different epitope towards developing a sandwich immunoassay not reliant on monoclonal or polyclonal conventional antibodies. The selection of such sdAb from this same library for the development of an all sdAb immunoassay is the next goal of this research effort.

\section{Conclusions}

In conclusion, the antigen recognition capabilities of the llama derived sdAb A3 were strong and specific for its target, SEB. The A3 sdAb was also found to be extremely heat resilient and to refold correctly following heat denaturation. These traits make it a valuable contribution to the current biodefense and public health arsenals of threat detection molecules. The availability of high affinity and recyclable recognition elements, such as A3, might also be used to increase the functional range of biosensors in the near future.

\section{Methods}

\section{Immunization Protocol and Serology}

An adult male llama housed at Triple J Farms (Bellingham, WA) was immunized with purified SEB toxoid (Toxin Technologies) intramuscularly followed by three boosters at three week intervals with $225 \mu \mathrm{g}$ of purified SEB toxoid. Sera was collected immediately before each inoculation and used to monitor the total IgG response against SEB. Three weeks after the final immunization $500 \mathrm{~mL}$ of whole blood and $50 \mathrm{~mL}$ of plasma was collected and used for lymphocyte isolation and serology. White blood cells were immediately isolated from the whole blood for RNA purification. Plasma was stored at $-80^{\circ} \mathrm{C}$ until used. Animal studies were approved by a separate review board through Triple J Farms and Kent Laboratories.

Total IgG responses to SEB toxin (Sigma Aldrich) and toxoid were measured over the course of the immunization series by direct ELISA. Briefly, plate wells were coated with $0.5 \mu \mathrm{g} / \mathrm{mL}$ of SEB toxin or toxoid overnight at $4^{\circ} \mathrm{C}$. The following morning wells were washed three times with phosphate buffered saline (PBS) followed by addition of $3 \%$ non-fat Milk in PBS (MPBS) and kept at $37^{\circ} \mathrm{C}$ for two hours. Afterward, wells were washed again three times with PBS followed by coating them with diluted sera (1:1000) for 1 hour at $37^{\circ} \mathrm{C}$. The wells were again washed three times with PBS and incubated with anti-llama IgG $(\mathrm{H}+\mathrm{L})$ horse radish peroxidase (HRP) Conjugated (Bethyl Laboratories) at 1:5000 for 1 hour. Finally, wells were washed three times with PBS before the addition of 


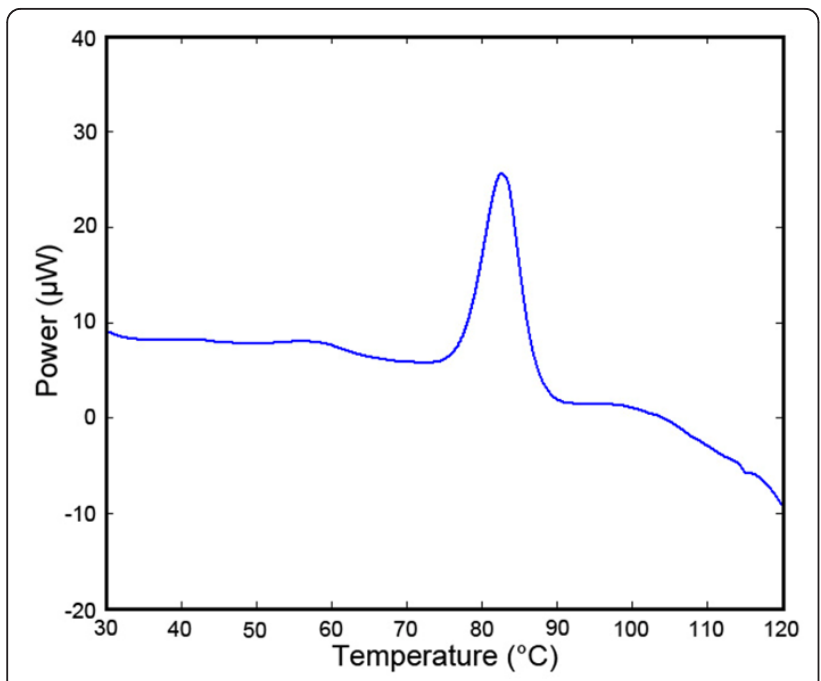

Figure 6 Differential Scanning Calorimetry of the sdAb A3. Antibody melting was observed in PBS with a protein concentration of $1.6 \mathrm{mg} / \mathrm{ml}$. Heating rate was $1^{\circ} \mathrm{C}$ per minute. Melting temperature was about $82.6^{\circ} \mathrm{C}$ and refolding upon cooling was not observed.

substrate reagent pack ( $\mathrm{R}$ and $\mathrm{D}$ Systems), and signal was read by optical density (OD) at 455 and $650 \mathrm{~nm}$.

\section{IgG antibody purification}

IgG was purified from llama plasma using a two-step process of Caprylic Acid (CA) to partially purify the IgG followed by using MEP HyperCel hydrophobic charge

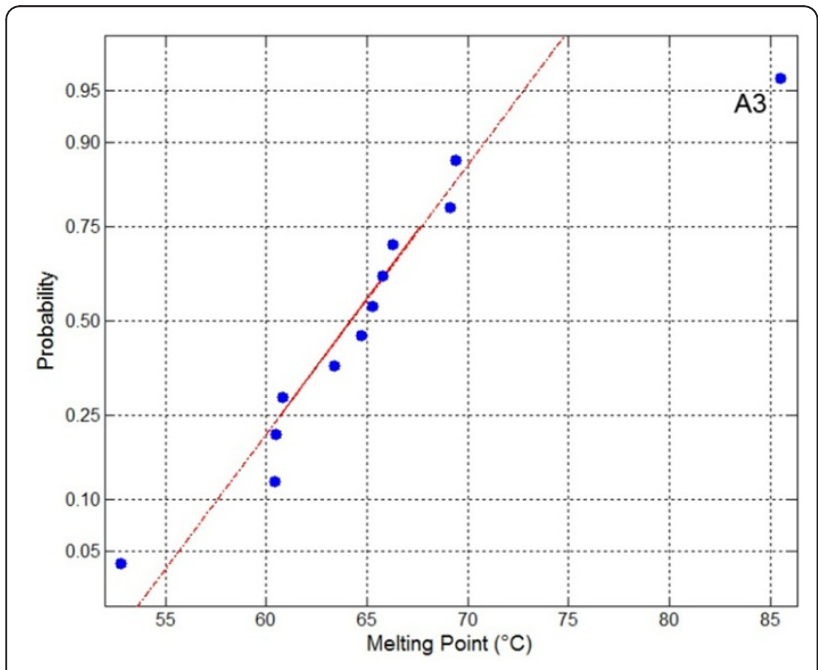

Figure 7 The $\mathrm{Tm}$ of $\mathrm{A} 3$ deviates from a normal distribution. A total of $12 \mathrm{SdAb}$ for which the melting temperatures have been determined are presented on a normal probability plot. The Y-axis values are specified by the probability distribution function for an assumed normal distribution. The straight line is a robust linear regression of the data and all data points will be near the line if the assumption is correct. The Tm for A3 can be seen to fit poorly. induction chromatography as previously described $[30,31]$. The IgG was separated into subclasses by a combination of affinity chromatography on protein $\mathrm{G}$ and protein A columns as described previously [32-34].

\section{Phagemid Library Construction and Panning}

White blood cells were isolated from the final post immunization bleed using Ficoll-Plaque Plus (GE Lifesciences), pelleted and washed with PBS, pelleted and stored at negative $80^{\circ} \mathrm{C}$ in Trizol (Invitrogen) until used for RNA extraction. RNA was extracted from Trizol and converted to cDNA using Arrayscript (Ambion) via oligo-dT primed reverse transcription. $\mathrm{V}_{\mathrm{HH}}$ coding regions were amplified by nested PCR as previously described [32,35]. The first round, $600 \mathrm{bp}$ amplicon, was agarose gel purified using UltrafreeDNA kit (Millipore) and used as the template for the secondary PCR reaction using the nested primers which amplify a $450 \mathrm{bp}$ region and incorporate SfiI and NotI restriction sites into the 5' and 3' ends, respectively [15]. The $450 \mathrm{bp}$ amplicons were agarose gel purified as before and then digested with SfiI and NotI. The resulting fragments were gel purified using Ultrafree-DNA system and concentrated with the Microcon YM-100 (Millipore). Purified DNA was ligated, using T4 DNA Ligase, into an open M13 phagemid vector, pCANTAB 5 E (GE Lifesciences). The ligations were then desalted and electroporated into electrocompetent XL-1 Blue Escherichia coli (Stratagene) and then pooled to form the library. A 10-fold serial dilution series of the library was plated out on Luria Broth plates containing $100 \mu \mathrm{g} / \mathrm{mL}$ Ampicillin (LB amp100) and colony counts used to estimate total library size of $\sim 10^{6}$ variants. Phage displayed SEB-specific $\mathrm{V}_{\mathrm{HH}}$ antibodies were isolated by phage rescue and panning. Phages were rescued via M13K07 phagemid purification [36]. Screened isolates were then used as the starting point for the second round of panning, and this process was repeated twice more for a total of 3 rounds of selective panning. Third round isolates were plaque assayed, randomly chosen from plates, to be screened for SEB-specific binding by ELISA. The plate was coated overnight with $5 \mu \mathrm{g} / \mathrm{mL}$ SEB in PBS. The wells were then blocked with 3\% MPBS for $2 \mathrm{hrs}$ at $37^{\circ} \mathrm{C}$. PEG precipitated phage from 96 individual colonies was added to each well, and the plate was incubated for 90 minutes at room temperature. AntiM13-HRP conjugate (GE Lifesciences) diluted 1:5000 in MPBS was added to each well and incubated for 90 min at room temperature. Plates were developed with the substrate reagent pack, and the reaction was monitored at OD 455 and $650 \mathrm{~nm}$. Clones were ranked based on absorbance and the top 11 chosen for further characterization. 

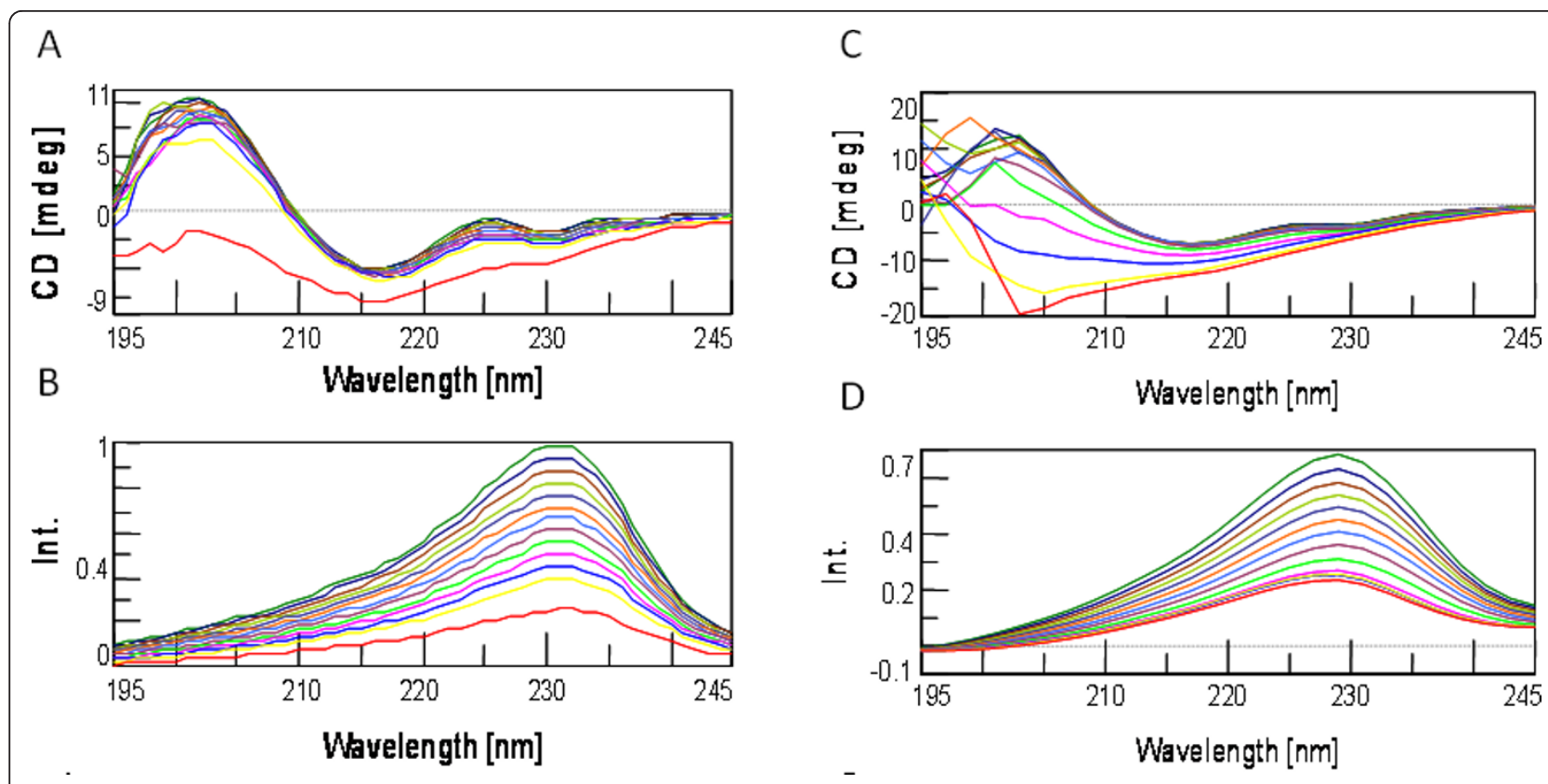

Figure 8 Comparison of $C D$ spectra and intrinsic fluorescence intensity of sdAb A3 to llama lgG1 taken at $5^{\circ} \mathrm{C}$ intervals from $25^{\circ} \mathrm{C}$ to $85^{\circ} \mathrm{C}$. SdAb A3 a) CD spectra b) fluorescence intensity. Llama anti SEB IgG1 c) CD spectra d) fluorescence intensity. Results indicate that intrinsic fluorescence changes fail to correlate with the sdAb's loss of ellipticity upon heating, while intrinsic fluorescence does correlate with ellipticity for the polyclonal lgG.

\section{Sequencing}

DNA sequences spanning the $\mathrm{V}_{\mathrm{HH}}$ portion of each of the 11 high binders were determined by commercial sequencing provided by MCLAB. Sequences were amplified using lacZ' forward primer 5'-ctatgaccatgattacgaatttctag-3'. Sequence analysis was performed using Geneious software (Biomatters Ltd.).

\section{Protein Expression and Purification}

A single sdAb variant, A3, was chosen for further analysis and cloned into the pecan 45 expression vector (kindly provided by Andrew Hayhurst, Southwest Foundation for Biomedical Research) and transformed into BL-21 Rosetta Escherichia coli cells (Novagen). SdAb protein was overexpressed and purified following protocols described previously [11]. In brief, A3 was overexpressed using Isopropyl $\beta$-D-1-thiogalactopyranoside (Fisherbrand); proteins were extracted from the periplasmic compartment by osmotic shock and purified by Immobilized Metal Ion Affinity Chromatography (IMAC) via incubation and elution from Nickel Sepharose (GE Lifesciences) [37]. Purified protein was stored at $4^{\circ} \mathrm{C}$ prior to gel filtration on a

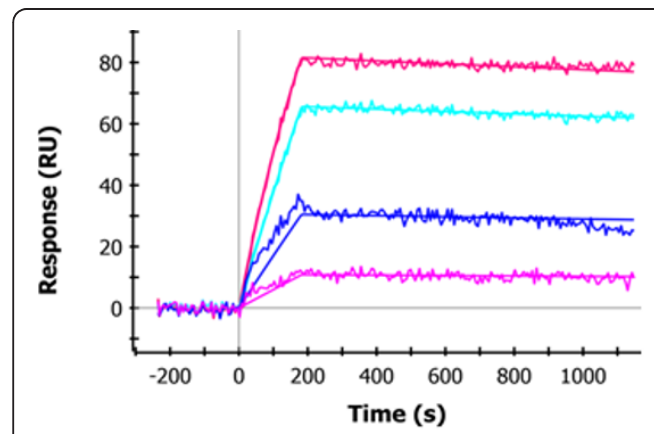

A3 sdab capture of SEB

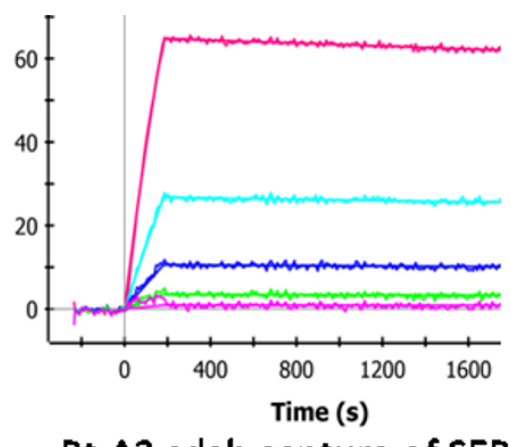

Bt-A3 sdab capture of SEB

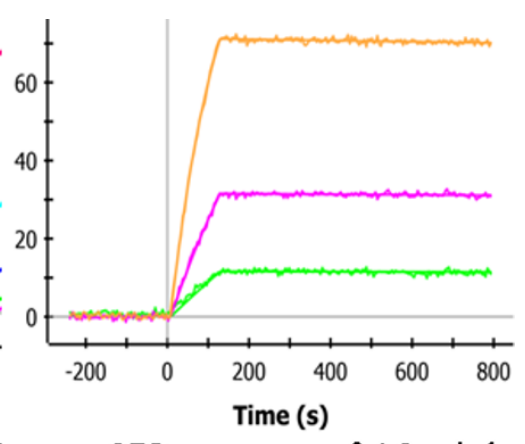

SEB capture of A3 sdab

Figure 9 SPR binding profiles for sdAb A3. Panel A shows five concentrations of SEB (30, 10, 3.3, 1.1, and 0.37 nM) flowed for 3 minutes over immobilized sdAb A3, followed by 15 minutes dissociaiton. Data shown was corrected by subtraction of interspot data and the buffer only response. Panel B shows a similar experiment performed on a NeutrAvidin coated chip with Bt-sdAb A3 immobilized. Panel C shows sdAb A3 $(30,10,3.3,1.1$, and $0.37 \mathrm{~nm}$ binding to a surface coated with SEB. 
Table 1 Result of SPR binding analysis

\begin{tabular}{cccc}
\hline Capture Molecule & $\begin{array}{c}\text { ka } \\
\mathbf{1 / M s}\end{array}$ & $\begin{array}{c}\text { kd } \\
\mathbf{1 / s}\end{array}$ & KD \\
\hline SdAb-A3 & $1.0 \times 10^{+5}$ & $6.0 \times 10^{-5}$ & $6.0 \times 10^{-10}$ \\
\hline NA-Bt-A3 & $7.2 \times 10^{+4}$ & $2.8 \times 10^{-5}$ & $3.9 \times 10^{-10}$ \\
\hline SEB & $2.9 \times 10^{+5}$ & $2.2 \times 10^{-5}$ & $7.5 \times 10^{-11}$ \\
\hline
\end{tabular}

The kinetic binding results obtained from the data shown in Figure 8. Kinetic parameters were determined by fitting the curves using a Langmuir model.

Superdex G75 column (GE Lifesciences) [11]. Protein was quantified using micro-BCA assay (Pierce) following manufacturer's protocols.

Western blots were preformed to confirm the purified protein's size. Purified protein samples were analyzed by SDS-PAGE on $12 \%$ Tris-Cl polyacrylamide gel by electrophoresis for 37 minutes at $200 \mathrm{~V}$. Following electrophoresis the gel was transferred to a nitrocellulose membrane (BioRad) for 1 hour at $100 \mathrm{~V}$. Membranes were incubated overnight at $4{ }^{\circ} \mathrm{C}$ with MPBS with $0.05 \%$ Tween 20 (MPBST), followed by incubation with anti-6 $\times$ His-HRP antibody (GenScript) for 1 hour at room temperature with shaking. The membranes were developed using Immuno-star Western C kits (Bio-Rad) following the manufacture's protocols, and images were captured on Versadoc (BioRad).

\section{Luminex Analysis}

A Luminex 100 flow analyzer was used to determine the specificity of the isolated sdAb as well as to perform sandwich assays for SEB. Antibodies were biotinylated (Bt) as previously described [25] using NHS-LC-biotin dissolved first in DMSO; a 20:1 NHS-biotin molar excess was used. Gel filtration on a Bio-gel P10 column was used to separate the biotin excess. Luminex microspheres for direct binding assays were coated with: two lots of SEB toxin, bovine albumin (BSA) (Sigma); Shiga Toxin 1 (STX-1), staphylococcal enterotoxin D (SED), staphylococcal enterotoxin A (SEA) (Toxin Technologies); Ricin, Ricinus communis Agglutinin 120 (RCA120) (Vector); Cholera Toxin (CTX) (Calbiochem). For sandwich immunoassays, microspheres were coated with the A3 sdAb, the purified polyclonal llama anti-SEB, and a monoclonal anti SEB MAb 3b2a kindly provided by Dr. Jill Czarnecki (Naval Medical Research Center, Silver Spring, MD). The protocol provided by Luminex for two-step carbodiimide coupling as previously described [11] was utilized to coat $0.1 \mathrm{~mL}$ of microspheres using $0.1 \mathrm{mg}$ protein at a concentration of $\sim 1$ $\mathrm{mg} / \mathrm{mL}$. The protein-coated microspheres were stored at $4^{\circ} \mathrm{C}$ in the dark until use.

Sandwich immunoassays for SEB were performed using A3 as both the capture molecule as well as the biotinylated detector In addition, the monoclonal
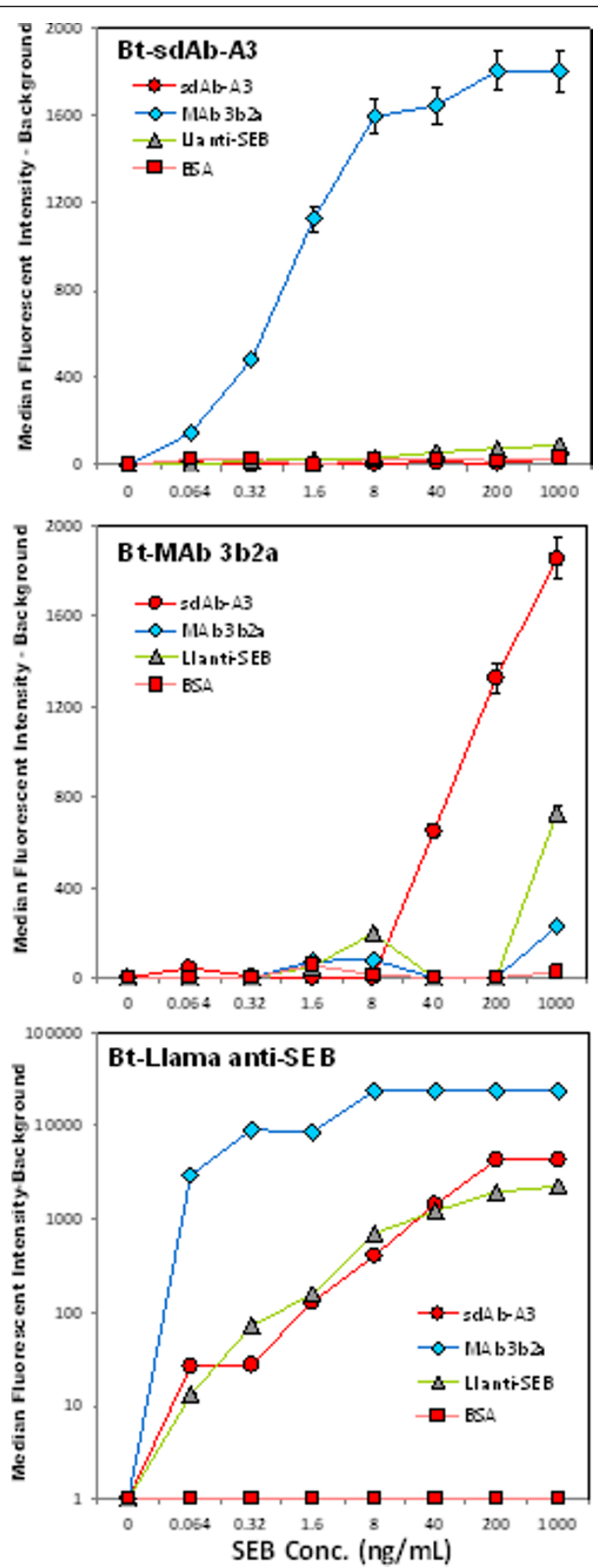

Figure 10 SEB detection using Luminex sandwich assays. Three different anti-SEB antibody coated bead sets plus a negative control were used in each assay performed as described in the methods. Top panel used Bt-sdAb-A3 $(1 \mu \mathrm{g} / \mathrm{mL})$, middle panel used Bt-MAb 3b2a $(10 \mu \mathrm{g} / \mathrm{mL})$, and bottom panel Bt-Llama anti-SEB $(10 \mu \mathrm{g} / \mathrm{mL})$, respectively as the detector antibody, followed by SA-PE $(5 \mu \mathrm{g} / \mathrm{mL})$ to fluorescently label the immuno-complex prior to measurement on the Luminex 100. 
antibody MAb 3b2a and the purified llama polyclonal anti-SEB (Ll-anti-SEB) antibody were also tested as the capture and detector molecule. SEB at a variety of concentrations was incubated with the antibody coated microspheres for 30 minutes. Unbound SEB was removed by filtration and the microspheres washed twice with PBS containing 0.05\% Tween-20 (PBST). The microspheres were then incubated with biotinylated detector antibodies: Bt-A3 (1 or $2 \mu \mathrm{g} / \mathrm{mL})$, Bt-Mab 3b2a $(5 \mu \mathrm{g} / \mathrm{mL}), \mathrm{Bt}-\mathrm{Ll}$-anti-SEB $(10 \mu \mathrm{g} / \mathrm{mL})$ for 30 minutes. After washing twice the amount bound was fluorescently labeled by the addition of $5 \mu \mathrm{g} / \mathrm{mL}$ SA-PE. After 30 minutes the excess SA-PE was removed and the microspheres resuspended in $85 \mu \mathrm{L}$ PBST and measured on a Luminex 100.

Thermal stability was tested using biotinylated antibodies (polyclonal llama anti-SEB IgG, MAb 3b2a, and sdAb A3 anti-SEB). The antibody samples were heated in a thermocycler at $85^{\circ} \mathrm{C}$ for different times up to 60 minutes, stored at $4^{\circ} \mathrm{C}$ for 1 hour, and then evaluated using a direct binding Luminex assay. Specificity of binding of the A3 variant was evaluated by a cross-reaction panel to various toxins and proteins (see above list) coupled to microspheres via a direct binding assay. Purified A3 sdAb was allowed to bind for 30 minutes at room temperature then the entire panel was run simultaneously on the Luminex.

\section{Circular Dichroism (CD)}

The melting point of the A3 sdAb was measured by circular dichroism using a Jasco J-815 CD spectropolarimeter equipped with a PTC-423S single position peltier temperature control system. Samples $(\sim 30 \mu \mathrm{g} / \mathrm{mL})$ were prepared by extensive dialysis versus $5 \mathrm{mM}$ sodium borate $\mathrm{pH} 7.5$, or by simple dilution with that same buffer. All measurements were made in a $10 \mathrm{~mm}$ path length quartz cuvette with a stir bar. The data were acquired from $245 \mathrm{~nm}$ to $195 \mathrm{~nm}$ at a scanning speed of $20 \mathrm{~nm} / \mathrm{min}$. The data pitch was $1 \mathrm{~nm}$, D.I.T. 2 seconds, band width $1 \mathrm{~nm}$, temperature ramp rate of $5^{\circ} \mathrm{C} / \mathrm{min}$ over the range of 25 to $95^{\circ} \mathrm{C}$. Intrinsic protein fluorescence intensity was also measured simultaneously using a $345 \mathrm{~nm}$ filter.

\section{Differential Scanning Calorimetry (DSC)}

The melting point was also measured by DSC using a TA Instruments NanoDSC. Antibody at a concentration of $1.6 \mathrm{mg} / \mathrm{ml}$ in PBS was scanned at a rate of $1^{\circ} \mathrm{C}$ per minute from $25^{\circ} \mathrm{C}$ to $120^{\circ} \mathrm{C}$.

\section{Surface Plasmon resonance (SPR) kinetics analysis}

The SPR kinetic measurements were performed using the ProteON (Bio-Rad). For testing the kinetics of the anti-SEB sdAb A3 a variety of surfaces were prepared.
In one case a GLC chip was coated with the sdAb A3 (5 $\mu \mathrm{g} / \mathrm{mL}$ ) along with sdAb specific for other targets (none of which bound SEB and served as negative controls for the tests presented here). A GLC chip was also coated with SEB $(5 \mu \mathrm{g} / \mathrm{mL})$, as well as other toxins which served as negative controls. All the protein samples to be immobilized were diluted in $10 \mathrm{mM}$ acetate buffer $\mathrm{pH} 5.0$ following the standard EDC coupling chemistry provided by the manufacturer. To ensure that biotinylation of the sdAb A3 had no affect on its activity, a NeutrAvidin coated chip was coated with Bt-sdAb A3. All experiments were performed at $25^{\circ} \mathrm{C}$. The binding of the sdAb were tested by flowing 6 concentrations varying from $30 \mathrm{nM}$ to $0 \mathrm{nM}$ for 180 seconds over the SEB coated chip, and then monitoring dissociation for 900 seconds. The chip was regenerated using $50 \mathrm{mM}$ glycine- $\mathrm{HCl}(\mathrm{pH} 2.0)$ for 36 seconds, prior to any additional testing. The data were analyzed with the ProteON Manager TM 2.1 software; binding constants were determined using the software's Langmuir model. Assays for binding of SEB by the immobilized sdAb were done in an analogous manner.

\section{Acknowledgements}

The authors would like to thank Dr. Andrew Hayhurst of Southwest Foundation for Biomedical Research for kindly providing the pecan 45 expression vector and Dr. Marla Swain for her helpful discussions. Our sincere thanks go to Fred Steinberg for his scientific guidance and critical review of this manuscript. This work was supported by the MITRE Innovation Program, JSTO-CBD/DTRA, and the Office of Naval Research NRL base funds. J.S. was supported by an NSF Historically Black Colleges and Universities Tribal College or University internship program.

\section{Author details}

'MITRE Corporation, 7515 Colshire Drive, McLean, Virginia, 22102, USA. ${ }^{2}$ Center for Bio/Molecular Science and Engineering, Naval Research Laboratory, 4555 Overlook Avenue SW, Washington, DC, 20375, USA. ${ }^{3}$ Department of Biology, University of Puerto Rico at Arecibo, Arecibo, 00613, Puerto Rico.

\section{Authors' contributions}

RRG developed the phage display library, selected the A3 binder, and produced the soluble protein and wrote the manuscript. GPA performed the SPR and Luminex 100 immunoassay testing and edited the manuscript. KAD titered the llama immune serum. DZ performed most of the CD and DSC experiments and evaluated TM to develop Figure 7. JLL assisted with the library development. JSG purified the IgG subclasses and tested the thermal stability by CD and fluorescence and Luminex 100. ERG devised experiments, managed the NRL efforts and edited the manuscript. LAC conceived of this project, managed the efforts at MITRE, and edited the manuscript. All authors read and approved the final manuscript.

\section{Competing interests}

The authors declare that they have no competing interests.

Received: 2 June 2011 Accepted: 21 September 2011 Published: 21 September 2011

\section{References}

1. Anthrax Documents. 2010 [http://www.justice.gov/amerithrax/, 2/15/2011 edn.

2. Leski TA, Lin B, Malanoski AP, Wang Z, Long NC, Meador CE, Barrows B, Ibrahim S, Hardick JP, Aitichou M, et al: Testing and Validation of High 
Density Resequencing Microarray for Broad Range Biothreat Agents Detection. Plos One 2009, 4(8).

3. Yang S, Rothman RE, Hardick J, Kuroki M, Hardick A, Doshi V, Ramachandran P, Gaydos CA: Rapid polymerase chain reaction-based screening assay for bacterial biothreat agents. Academic Emergency Medicine 2008, 15(4):388-392.

4. Lim DV, Simpson JM, Kearns EA, Kramer MF: Current and developing technologies for monitoring agents of bioterrorism and biowarfare. Clinical Microbiology Reviews 2005, 18(4):583, +.

5. Ngom B, Guo YC, Wang XL, Bi DR: Development and application of lateral flow test strip technology for detection of infectious agents and chemical contaminants: a review. Analytical and Bioanalytical Chemistry 2010, 397(3):1113-1135.

6. Posthuma-Trumpie GA, Korf J, van Amerongen A: Lateral flow (immuno) assay: its strengths, weaknesses, opportunities and threats. A literature survey. Analytical and Bioanalytical Chemistry 2009, 393(2):569-582

7. McAteer K, Simpson CE, Gibson TD, Gueguen S, Boujtita M, El Murr N: Proposed model for shelf-life prediction of stabilised commercial enzyme-based systems and biosensors. J Mol Catal B-Enzym 1999, 7(14):47-56.

8. Leuvering JHW, Thal P: Colloidal gold particles coated with monoclonalantibodies-preparation and shelf-life. Ultramicroscopy 1984, 14(4):412-413.

9. Hamers-Casterman C, Atarhouch T, Muyldermans S, Robinson G, Hamers C, Songa EB, Bendahman N, Hamers R: Naturally-Occurring Antibodies Devoid of Light-Chains. Nature 1993, 363(6428):446-448.

10. Bird RE, Hardman KD, Jacobson JW, Johnson S, Kaufman BM, Lee SM, Lee T, Pope SH, Riordan GS, Whitlow M: Single-chain antigen-binding proteins. Science 1988, 242(4877):423-426.

11. Goldman ER, Anderson GP, Liu JL, Delehanty JB, Sherwood LJ, Osborn LE, Cummins LB, Hayhurst A: Facile generation of heat-stable antiviral and antitoxin single domain antibodies from a semisynthetic llama library. Analytical Chemistry 2006, 78(24):8245-8255.

12. van der Linden RHJ, Frenken LGJ, de Geus B, Harmsen MM, Ruuls RC, Stok W, de Ron L, Wilson S, Davis P, Verrips CT: Comparison of physical chemical properties of llama V-HH antibody fragments and mouse monoclonal antibodies. Biochim Biophys Acta-Protein Struct Molec Enzym 1999, 1431(1):37-46.

13. Wesolowski J, Alzogaray V, Reyelt J, Unger M, Juarez K, Urrutia M, Cauerhff A, Danquah W, Rissiek B, Scheuplein F, et al: Single domain antibodies: promising experimental and therapeutic tools in infection and immunity. Medical Microbiology and Immunology 2009, 198(3):157-174.

14. Goldman ER, Anderson GP, Conway J, Sherwood LJ, Fech M, Vo B, Liu JL, Hayhurst A: Thermostable Llama Single Domain Antibodies for Detection of Botulinum A Neurotoxin Complex. Analytical Chemistry 2008, 80(22):8583-8591.

15. Ladenson RC, Crimmins DL, Landt Y, Ladenson JH: Isolation and characterization of a thermally stable recombinant anti-caffeine heavychain antibody fragment. Analytical Chemistry 2006, 78(13):4501-4508

16. Alvarez-Rueda N, Behar G, Ferre V, Pugniere M, Roquet F, Gastinel L, Jacquot C, Aubry J, Baty D, Barbet J, et al: Generation of llama singledomain antibodies against methotrexate, a prototypical hapten. Molecular Immunology 2007, 44(7):1680-1690.

17. Sherwood L, Osborn LE, Carrion R, Patterson JL, Hayhurst A: Rapid assembly of sensitive antigen-capture assays for Marburg virus, using in vitro selection of llama single-domain antibodies, at biosafety level 4. J Infect Dis 2007, 196:S213-S219.

18. Bouhaouala-Zahar B, Hmila I, Saerens D, Ben Abderrazek R, Vincke C, Abidi N, Benlasfar Z, Govaert J, El Ayeb M, Muyldermans S: A bispecific nanobody to provide full protection against lethal scorpion envenoming. Faseb Journal 2010, 24(9):3479-3489.

19. Pant $N$, Marcotte $H$, Hermans $P$, Bezemer $S$, Frenken $L$, Johansen $K$, Hammarstrom L: Lactobacilli producing bispecific llama-derived antirotavirus proteins in vivo for rotavirus-induced diarrhea. Future Microbiology 2011, 6(5):583-593.

20. Verrips T, Koh WWL, Steffensen S, Gonzalez-Pajuelo M, Hoorelbeke B, Gorlani A, Szynol A, Forsman A, Aasa-Chapman MMl, de Haard H, et al: Generation of a Family-specific Phage Library of Llama Single Chain Antibody Fragments That Neutralize HIV-1. J Biol Chem 2010, 285(25):19116-19124

21. Adams H, Brummelhuis W, Maassen B, van Egmond N, El Khattabi M, Detmers F, Hermans P, Braam B, Stam J, Verrips T: Specific immuno capturing of the staphylococcal superantigen toxic-shock syndrome toxin-1 in plasma. Biotechnol Bioeng 2009, 104(1):143-151.

22. Ryan S, Kell AJ, van Faassen H, Tay LL, Simard B, Mackenzie R, Gilbert M, Tanha J: Single-Domain Antibody-Nanoparticles: Promising Architectures for Increased Staphylococcus aureus Detection Specificity and Sensitivity. Bioconjugate Chemistry 2009, 20(10):1966-1974.

23. Huang IY, Bergdoll MS: The primary structure of staphylococcal enterotoxin B. 3. The cyanogen bromide peptides of reduced and aminoethylated enterotoxin $\mathrm{B}$, and the complete amino acid sequence. J Biol Chem 1970, 245(14):3518-3525.

24. Swain MD, Anderson GP, Zabetakis D, Bernstein RD, Liu JL, Sherwood LJ, Hayhurst A, Goldman ER: Llama-derived single-domain antibodies for the detection of botulinum A neurotoxin. Analytical and Bioanalytical Chemistry 2010, 398(1):339-348.

25. Anderson GP, Liu JL, Hale ML, Bernstein RD, Moore M, Swain MD, Goldman ER: Development of Antiricin Single Domain Antibodies Toward Detection and Therapeutic Reagents. Analytical Chemistry 2008, 80(24):9604-9611.

26. Conway JO, Sherwood LJ, Collazo MT, Garza JA, Hayhurst A: Llama Single Domain Antibodies Specific for the 7 Botulinum Neurotoxin Serotypes as Heptaplex Immunoreagents. Plos One 2010, 5:(1).

27. Perez JMJ, Renisio JG, Prompers JJ, van Platerink CJ, Cambillau C, Darbon H, Frenken LGJ: Thermal unfolding of a llama antibody fragment: A twostate reversible process. Biochemistry 2001, 40(1):74-83.

28. Dumoulin M, Conrath K, Van Meirhaeghe A, Meersman F, Heremans K Frenken LGJ, Muyldermans S, Wyns L, Matagne A: Single-domain antibody fragments with high conformational stability. Protein Science 2002, 11(3):500-515

29. Davies J, Riechmann L: Antibody Vh Domains as Small Recognition Units. Bio-Technology 1995, 13(5):475-479.

30. McLaren RD, Prosser CG, Grieve RC, Borissenko M: The use of caprylic acid for the extraction of the immunoglobulin fraction from egg yolk of chickens immunised with ovine alpha-lactalbumin. J Immunol Methods 1994, 177(1-2):175-184.

31. Anderson GP, Ortiz-Vera YA, Czarnecki J, BaoHan Vo B, ER G: Evaluation of Ilama anti-botulinum toxin polyclonal IgG subclasses. The Botulinum Journal 2008, 1:100-115.

32. van der Linden RHJ, de Geus B, Frenken LGJ, Peters H, Verrips CT: Improved production and function of llama heavy chain antibody fragments by molecular evolution. Journal of Biotechnology 2000, 80(3):261-270.

33. Meddeb-Mouelhi F, Bouhaouala-Zahar B, Benlasfar Z, Hammadi M, Mejri T, Moslah M, Karoui H, Khorchani T, El Ayeb M: Immunized camel sera and derived immunoglobulin subclasses neutralizing Androctonus australis hector scorpion toxins. Toxicon 2003, 42(7):785-791.

34. Daley LP, Gagliardo LF, Duffy MS, Smith MC, Appleton JA: Application of monoclonal antibodies in functional and comparative investigations of heavy-chain immunoglobulins in new world camelids. Clin Diagn Lab Immunol 2005, 12(3):380-386.

35. Arbabi Ghahroudi M, Desmyter A, Wyns L, Hamers R, Muyldermans S: Selection and identification of single domain antibody fragments from camel heavy-chain antibodies. FEBS Letters 1997, 414(3):521-526.

36. Griffiths AD, Malmqvist M, Marks JD, Bye JM, Embleton MJ, McCafferty J, Baier M, Holliger KP, Gorick BD, Hughes-Jones NC, et al: Human anti-self antibodies with high specificity from phage display libraries. EMBO J 1993, 12(2):725-734.

37. Hayhurst A, Happe S, Mabry R, Koch Z, Iverson BL, Georgiou G: Isolation and expression of recombinant antibody fragments to the biological warfare pathogen Brucella melitensis. Journal of Immunological Methods 2003, 276(1-2):185-196

doi:10.1186/1472-6750-11-86

Cite this article as: Graef et al:: Isolation of a Highly Thermal Stable Lama Single Domain Antibody Specific for Staphylococcus aureus Enterotoxin B. BMC Biotechnology 2011 11:86 